\title{
Efficacy of percutaneous radiofrequency ablation for the treatment of hepatocellular carcinoma
}

\author{
R.N. Zheng ${ }^{1,2 *}$, Z.J. You ${ }^{2 *}$ S.H. Lin ${ }^{2 *}$, J. Jia², Y.M. Cai ${ }^{2}$, C. Liu $^{2}$, S. Han $^{3}$ and \\ S.M. Wang ${ }^{2}$ \\ 1Department of Oncology, Dongguan People's Hospital, Dongguan, China \\ ${ }^{2}$ Department of Oncology, Zhujiang Hospital, Southern Medical University, \\ Guangzhou, China \\ ${ }^{3}$ Department of General Surgery, Zhujiang Hospital, \\ Southern Medical University, Guangzhou, China \\ *These authors contributions equally to this study. \\ Corresponding author: S.M. Wang \\ E-mail: wangsenming0808@163.com
}

Genet. Mol. Res. 14 (4): 17982-17994 (2015)

Received August 20, 2015

Accepted October 9, 2015

Published December 22, 2015

DOI http://dx.doi.org/10.4238/2015.December.22.24

\begin{abstract}
In this study, we sought to evaluate the efficacy of transcatheter arterial chemoembolization (TACE) plus radiofrequency ablation (RFA; experimental group) versus RFA treatment (control group) in patients receiving palliative treatment for hepatocellular carcinoma. To summarize the available evidence, we used the Review Manager 5.1 software to perform a meta-analysis of English-language articles published in public databases prior to 2014. Based on 6 studies that met the inclusion criteria, a total of 531 (experimental group, 272; control group, 259) patients with hepatocellular carcinoma were included in the metaanalysis. The meta-analysis demonstrated that the experimental group had a higher 3-year survival rate [risk ratios $(\mathrm{RRs})=1.41 ; 95 \%$ confidence interval $(\mathrm{Cl})=1.03-1.94 ; \mathrm{P}<0.05]$ and a higher 2 -year survival rate (RR $=1.11 ; 95 \% \mathrm{Cl}=1.01-1.23 ; \mathrm{P}<0.05)$ than the control group. In the overall
\end{abstract}


meta-analysis, the overall RRs were $2.02(95 \% \mathrm{Cl}=1.40-2.91 ; \mathrm{P}<0.05)$ and $1.63(95 \% \mathrm{Cl}=1.06-2.51 ; \mathrm{P}<0.05)$ for 3 - and 5 -year recurrence-free survival, respectively. Furthermore, the overall meta-analysis showed an overall RR of $0.75(95 \% \mathrm{Cl}=0.60-0.93 ; \mathrm{P}<0.05)$ for the incidence of tumor progression and an overall RR of $1.19(95 \% \mathrm{Cl}=0.33-4.33 ; \mathrm{P}>0.05)$ for the major complication rate. In a sensitivity analysis, the above mentioned meta-analytic estimates were unchanged by the removal of 1 study at a time. The meta-analysis suggested that the experimental group had a higher survival rate, a higher recurrence-free survival rate, and a lower incidence of tumor progression than the corresponding control group.

Key words: TACE; RFA; Hepatocellular carcinoma; Meta-analysis

\section{INTRODUCTION}

Hepatocellular carcinoma is one of the most common cancers around the world (Archambeaud et al., 2014; Chang et al., 2014; Hallager et al., 2014; Ridruejo, 2014; Flemming et al., 2015; Li et al., 2015; Pais et al., 2015; Zhao et al., 2015); it is the sixth most common cancer and the third most common cause of cancer mortality worldwide (Zhang et al., 2015). There is a clear need for additional treatment options for hepatocellular carcinoma (Yang et al., 2008). Liver transplantation offers the most effective treatment for patients with hepatocellular carcinoma (Ferreiro et al., 2014). The prognosis of patients with intrahepatic cholangiocarcinoma is currently unsatisfactory (Wu et al., 2012). Transcatheter arterial chemoembolization (TACE), a procedure for the treatment of hepatocellular carcinoma, induces a pronounced but transient increase in hepatic cytolysis parameters (Bianco et al., 1996), and it has been widely used for inoperable hepatocellular carcinoma (Shimohira et al., 2011).

TACE combined with radiofrequency ablation (RFA) therapy has been used for patients with large hepatocellular carcinoma tumors (Cheng et al., 2008), but the survival benefits of combined treatment are not known. We conducted a meta-analysis to assess the survival rate, recurrence-free survival rate, incidence of tumor progression, and major complication rate for patients with hepatocellular carcinoma who were treated in an experimental or control group. With the ultimate goal of improving the efficacy of RFA for the treatment of hepatocellular carcinoma, we compared RFA plus TACE with RFA alone.

\section{MATERIAL AND METHODS}

\section{Source of material}

The following public databases were searched for articles published before December 2014: PubMed, MEDLINE, EMBASE, Springer, Elsevier Science Direct, the Cochrane Library, and Google Scholar. The key search terms included: "radiofrequency ablation", "RFA", "hepatocellular", "hepatic", "hepatoma", "liver cancer", "transcatheter arterial chemoembolization", "TACE", "efficacy", "study", and "trial". Concurrently, references from retrieved papers were checked for any additional studies. We only used data from fully published papers, and therefore excluded meeting and conference abstracts. 
This study was approved by the Ethics Committee of Dongguan People's Hospital.

\section{Criteria for study inclusion and exclusion}

The inclusion criteria for the studies were that they mainly involved investigations of patients with hepatocellular carcinoma and were randomized controlled trials (RCTs). Patients with hepatocellular carcinoma (the age range was not limited) and therapy including TACE plus RFA (experimental group) versus RFA (control group) were assessed for English-language papers, while effect sizes were evaluated in terms of odds ratios. No limits were placed on the sample sizes. For a study to be included, the study period had to end before 2014 and the publication year had to be prior to 2014. We excluded studies that only described RFA data, as well as reviews and case reports of patients with hepatocellular carcinoma, duplicate studies and records, and studies that did not compare experimental and control groups.

\section{Data extraction}

We conducted the data extraction after a research training exercise. Data items included study details (e.g., first author's name, study period, year of publication, location of participants, and method of study), characteristics of participants (e.g., sample size, age, and gender), and tumor sizes. Two investigators ( $A$ and $D$ ) extracted the data independently using a standard protocol, and the result was reviewed by a third investigator $(B)$. We contacted the authors of the included studies to obtain additional information for data items that needed clarification. Discrepancies were resolved through discussion with our research team or contact with the original investigators, who were all sent data extraction sheets with requests for correction. We recorded details including first author's name, publication year, country, sample size, gender, age, and tumor size.

\section{Statistical analysis}

The meta-analysis compared the risk ratios (RRs) for patients with hepatocellular carcinoma in the experimental and control groups. The overall or pooled estimate of RRs was obtained using the Mantel-Haenszel method in a fixed-effect model (Mantel and Haenszel, 1959) or the DerSimonian and Laid method in a random-effect model (DerSimonian and Laird, 1986). We assessed the withinand between-study variation or heterogeneity by testing Cochran's Q-statistic (Lau et al., 1997). We also quantified the effect of heterogeneity using $I^{2}=100 \% \times(Q-$ d.f.) / $Q$ (Higgins et al., 2003). A significant Q-statistic $(P<0.10)$ or $\mathrm{I}^{2}$-statistic $\left(\mathrm{I}^{2}>50 \%\right)$ indicated heterogeneity across studies, in which case the random-effect model was used for the meta-analysis. Otherwise, the fixed-effect model was used. The significance of the pooled RRs was determined using the Z-test.

The meta-analysis was performed using the Review Manager 5.1 software (Cochrane Collaboration, http://ims.cochrane.org/revman). All P values were two-sided. Although a $\mathrm{P}$ value of $<0.05$ was considered to be significant in the main analyses, $\mathrm{P}<0.1$ was considered significant in the heterogeneity analysis.

\section{Evaluation of publication bias}

We evaluated publication bias using a funnel plot. The funnel plot showed symmetry, indicating that no publication bias existed. 
Analyses were performed using the Review Manager 5.1 software (Cochrane Collaboration, http://ims.cochrane.org/revman) and the STATA software package v.11.0 (Stata Corporation, College Station, TX, USA). All P values were two sided. A P value of $<0.05$ was considered to be significant. In order to test the reliability of results, we also conducted a sensitivity analysis. In this sensitivity analysis, each study was removed in turn and the meta-analysis was repeated in its absence.

\section{RESULTS}

\section{Characteristics of eligible studies}

A total of 575 papers were relevant to the search terms (PubMed, 159; MEDLINE, 94; Springer, 108; Elsevier Science Direct, 55; Cochrane Library, 6; Google Scholar, 153). A flow diagram of the selection of studies is shown in Figure 1. There were 54 relevant studies after duplicates had been removed. While screening the abstracts, 29 of these articles were excluded (16 were review articles; 13 did not comprise hepatocellular carcinoma patients). Subsequently, 25 studies remained for a full-publication review, 19 of which were ultimately excluded (11 only reported RFA and did not conduct a comparison; 8 did not involve RCT).

A total of 6 studies were included in the meta-analysis. The characteristics of the examined studies are presented in Table 1. The included studies occurred between 2005 and 2012. A total of 531 (experimental group, 272; control group, 259) patients with hepatocellular carcinoma were considered in this meta-analysis. The patients' mean ages ranged from 55.1 to 69.8 years, the sample sizes ranged from 34 to 196, and mean tumor sizes ranged from 1.6 to $6.6 \mathrm{~cm}$.

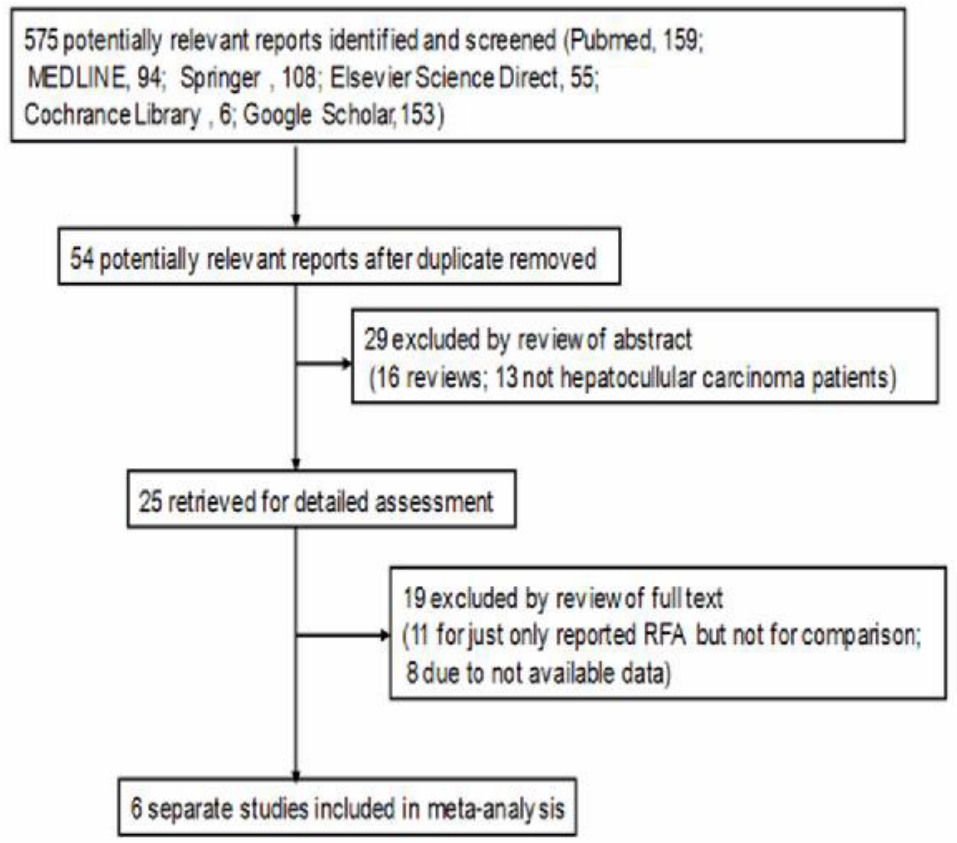

Figure 1. Flow diagram of the selection of studies. 
R.N. Zheng et al.

17986

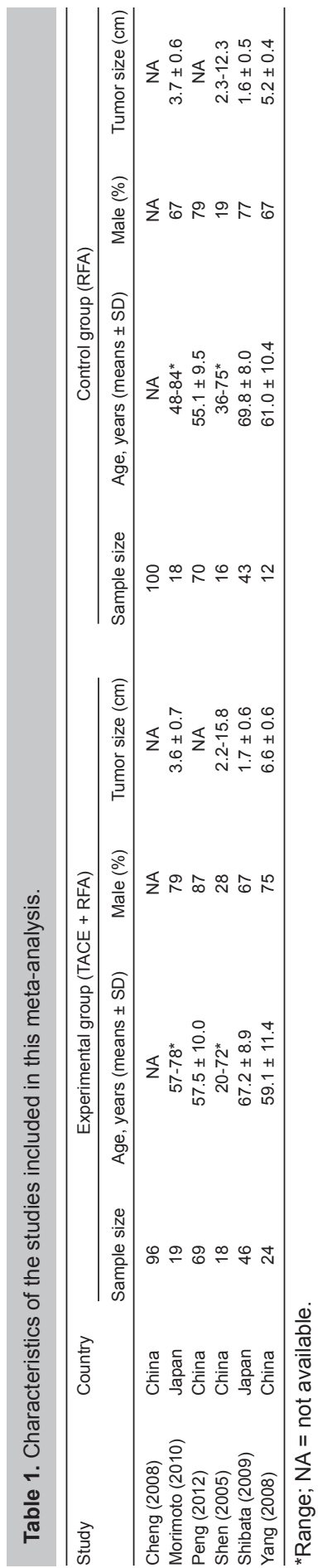

Genetics and Molecular Research 14 (4): 17982-17994 (2015) 


\section{Overall effects on survival rate in experimental and control groups}

As shown in Figure 2, the meta-analysis of survival rates included 6 separate studies (Shen et al., 2005; Yang et al., 2008; Cheng et al., 2008; Shibata et al., 2009; Morimoto et al., 2010; Peng et al., 2012), comprising 531 (experimental group, 272; control group, 259) patients with hepatocellular carcinoma. The heterogeneity test did not indicate any significant heterogeneity among the studies $\left(Q^{2}=2.06 ; I^{2}=3.0 \% ; P>0.1\right)$. We therefore used a fixedeffects model to compare the survival rates in the experimental and control groups. Comparing the experimental and control groups, the overall meta-analysis showed overall RRs of 1.41 $(95 \% \mathrm{Cl}=1.03-1.94 ; \mathrm{P}<0.05)$ for the 3 -year survival rate and $1.11(95 \% \mathrm{Cl}=1.01-1.23 ; \mathrm{P}$ $<0.05$ ) for the 2-year survival rate, demonstrating that the experimental group had a higher 1-year survival rate than the control group. A funnel plot (Figure 3) showed no evidence of publication bias.

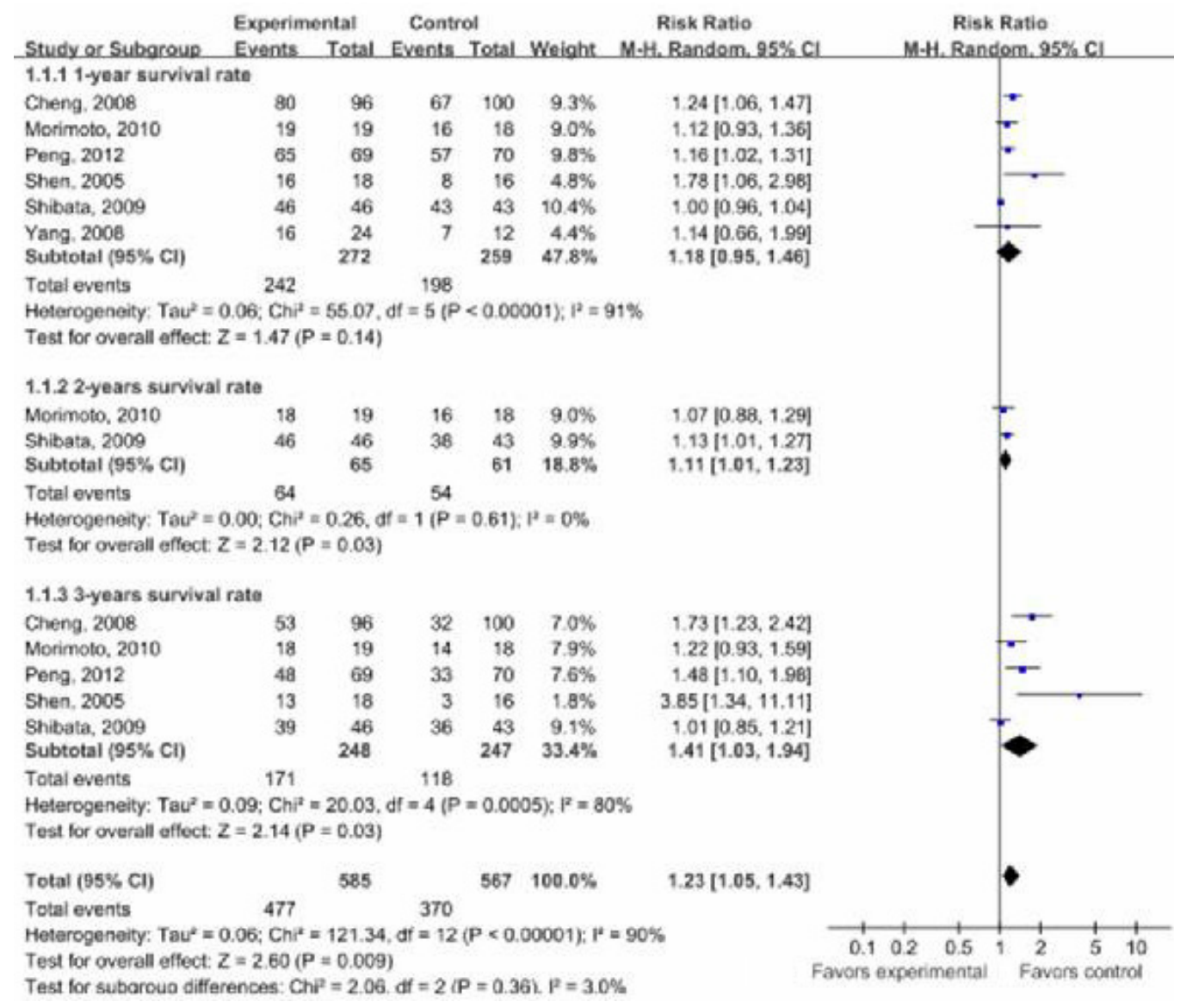

Figure 2. Forest plot of survival rates for the experimental groups versus the control groups of the studies included. 


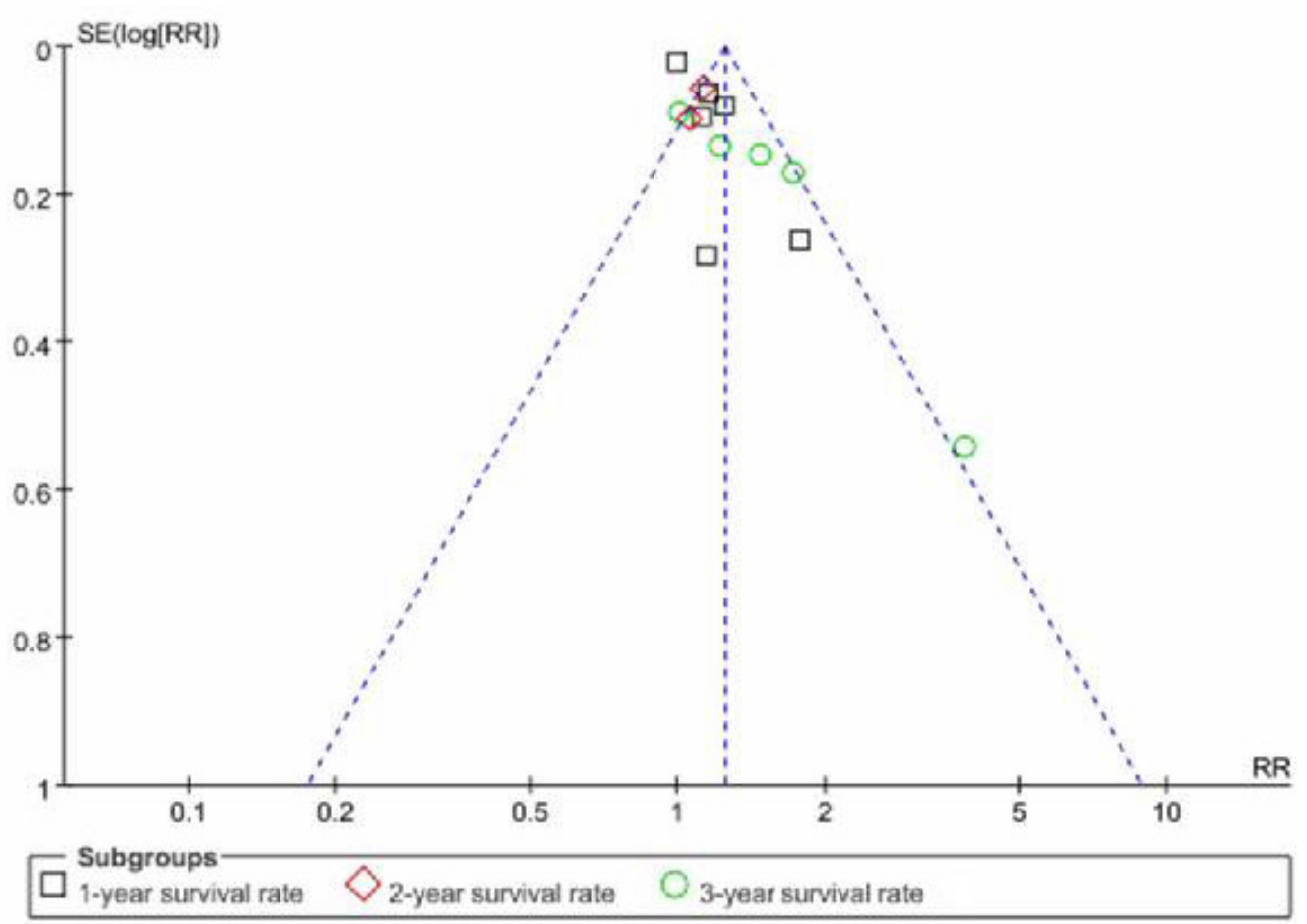

Figure 3. Funnel plot of survival rates for the experimental groups versus the control groups of the included studies.

\section{Overall effects on recurrence-free survival in experimental and control groups}

As shown in Figure 4, the meta-analysis of recurrence-free survival rates included a total of 6 separate studies (Shen et al., 2005; Yang et al., 2008; Cheng et al., 2008; Shibata et al., 2009; Morimoto et al., 2010; Peng et al., 2012), comprising 531 (experimental group, 272; control group, 259) patients with hepatocellular carcinoma. A heterogeneity test showed significant heterogeneity among the studies $\left(Q^{2}=8077 ; I^{2}=77.2 \% ; P<0.1\right)$. We therefore used a random-effects model to compare the recurrence-free survival rates of the experimental and control groups. The overall meta-analysis indicated that the overall RRs were $2.02(95 \% \mathrm{Cl}=1.40-2.91 ; \mathrm{P}<0.05)$ for the 3-year recurrence-free survival rate and $1.63(95 \% \mathrm{Cl}=1.06-2.51 ; \mathrm{P}<0.05)$ for the 5 -year recurrencefree survival rate in the experimental group versus the control group, demonstrating that the experimental group had higher 3- and 5-year recurrence-free survival rates than the control group. A funnel plot (Figure 5) showed no evidence of publication bias.

\section{Overall effects on the incidence of tumor progression in experimental and control groups}

As shown in Figure 6, the meta-analysis of the incidence of tumor progression included a total of 6 separate studies (Shen et al., 2005; Yang et al., 2008; Cheng et al., 2008; Shibata et al., 2009; Morimoto et al., 2010; Peng et al., 2012), comprising 531 (experimental group, 272; 
control group, 259) patients with hepatocellular carcinoma. The heterogeneity test did not show any significant heterogeneity among the studies $\left(Q^{2}=5.33 ; I^{2}=6.0 \% ; P>0.1\right)$. We therefore used a fixed-effects model to compare the incidences of tumor progression in the experimental and control groups. The overall meta-analysis indicated that the overall RRs were $0.75(95 \% \mathrm{Cl}=0.60-0.93 ; \mathrm{P}$ $<0.05$ ) for the incidence of tumor progression for patients in the experimental and control groups. These findings demonstrated that the experimental group had a lower incidence of tumor progression than the control group. A funnel plot (Figure 7) showed now evidence of publication bias.

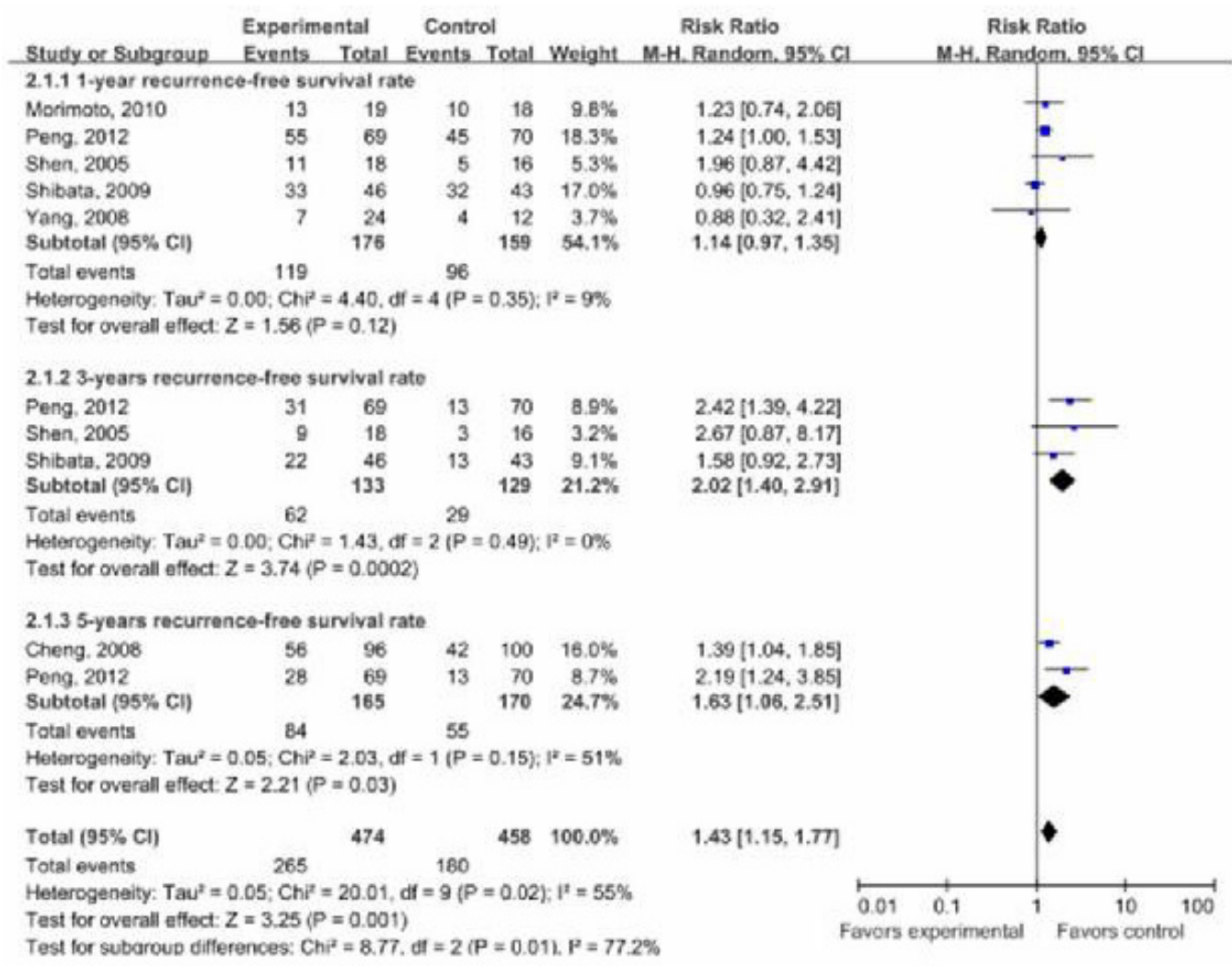

Figure 4. Forest plot of recurrence-free survival rates for the experimental groups versus the control groups of the studies included.

\section{Overall effects on the major complication rate in experimental and control groups}

As shown in Figure 8, the meta-analysis of the major complication rate included 3 separate studies (Shen et al., 2005; Shibata et al., 2009; Peng et al., 2012), comprising 262 (experimental group, 133; control group, 129) patients with hepatocellular carcinoma. The heterogeneity test did not show any heterogeneity among the studies $\left(Q^{2}=0.17 ; I^{2}=0 \% ; P>0.1\right)$. We therefore used the fixed-effects model to combine the major complication rates of the experimental and control 
groups. The overall meta-analysis indicated that the overall RRs were $1.19(95 \% \mathrm{Cl}=0.33-4.33$; $\mathrm{P}$ $>0.05$ ) for the major complication rate in the experimental group versus the control group. These findings indicate that the major complication rate for the experimental group was equal to that of the control group. A funnel plot (Figure 9) showed no evidence of publication bias.

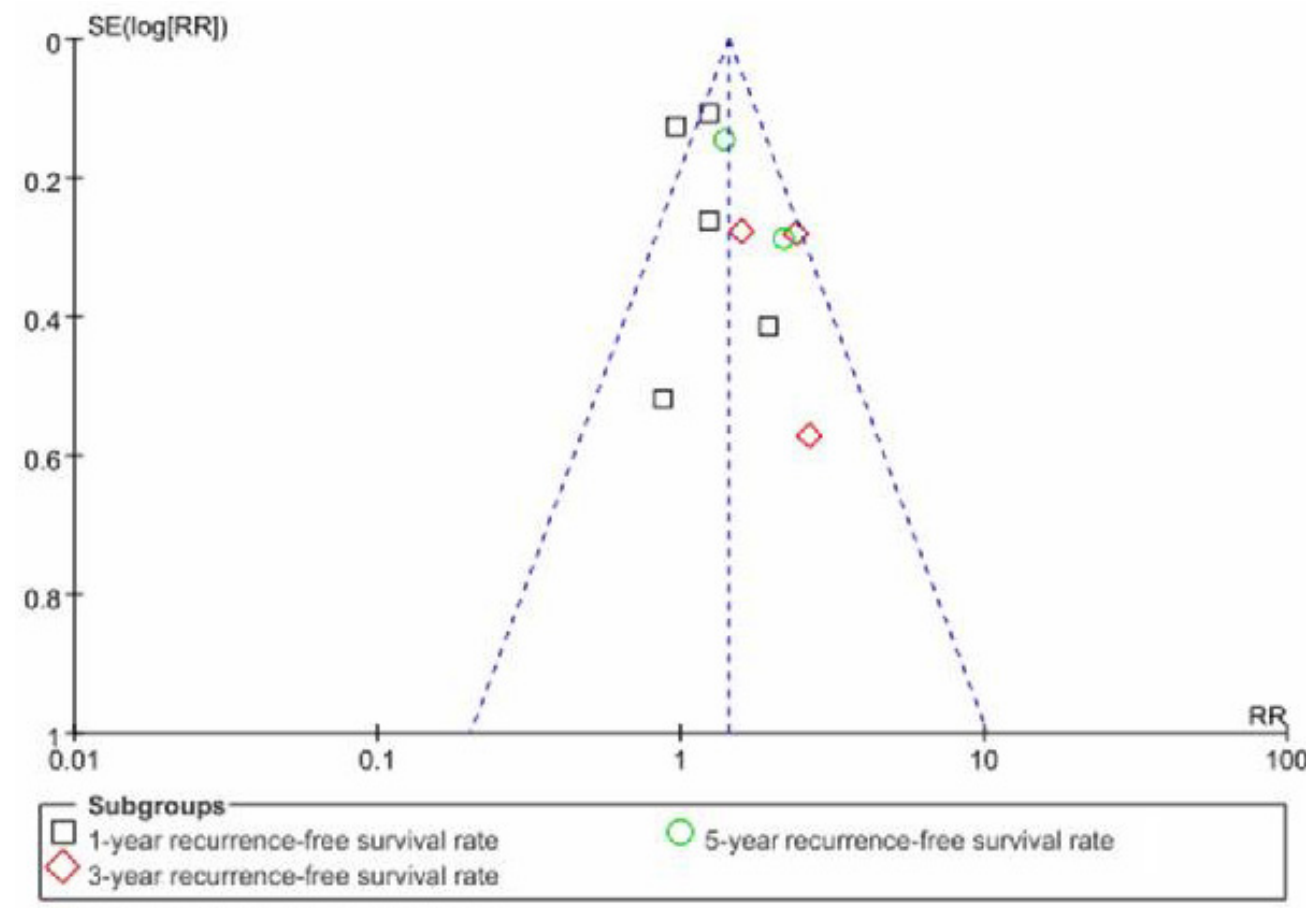

Figure 5. Funnel plot of recurrence-free survival rates for the experimental groups versus the control groups of the studies included.

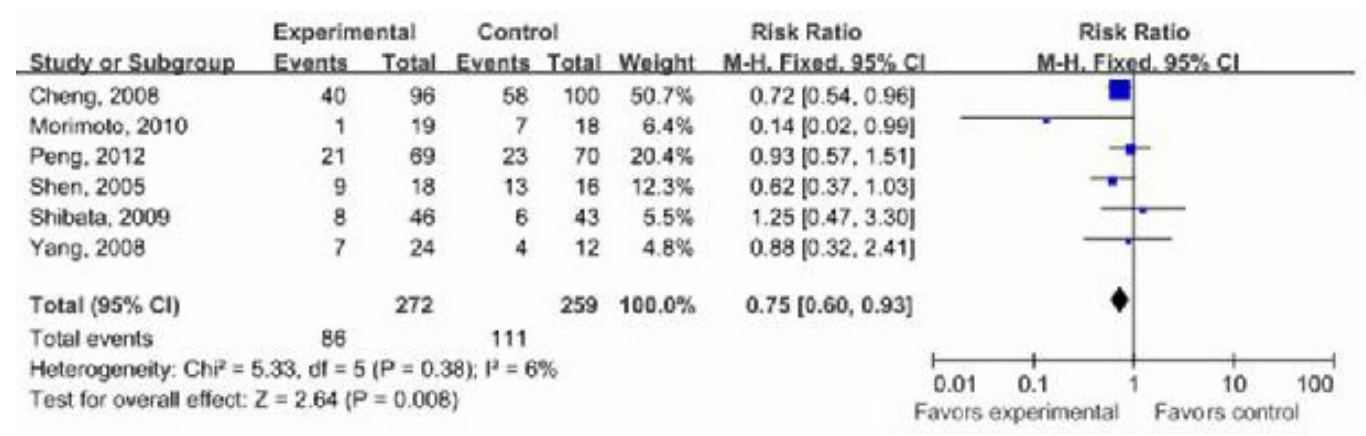

Figure 6. Forest plot of the incidence of tumor progression in the experimental groups versus the control groups of the studies included. 


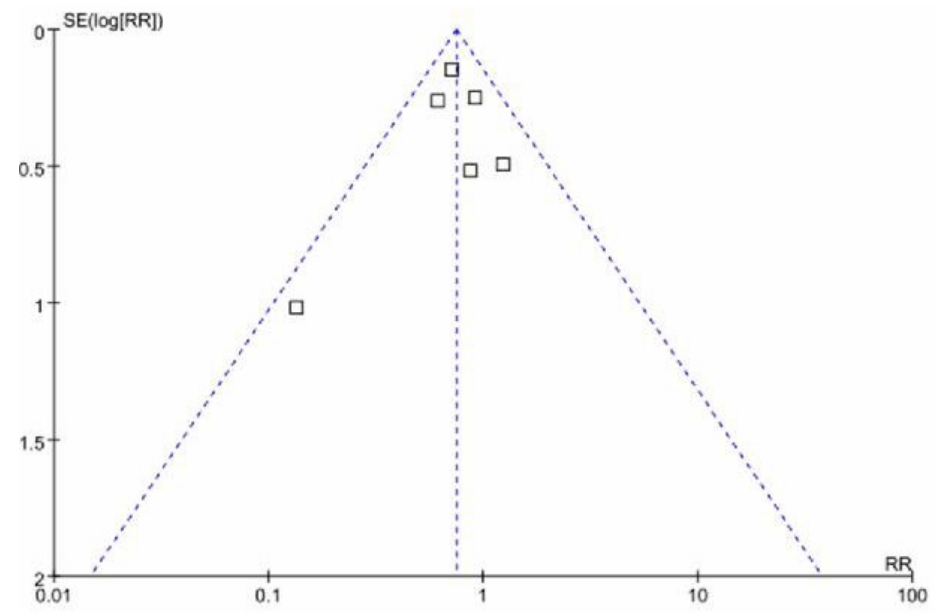

Figure 7. Funnel plot of the incidence of tumor progression in the experimental groups versus the control groups of the studies included.

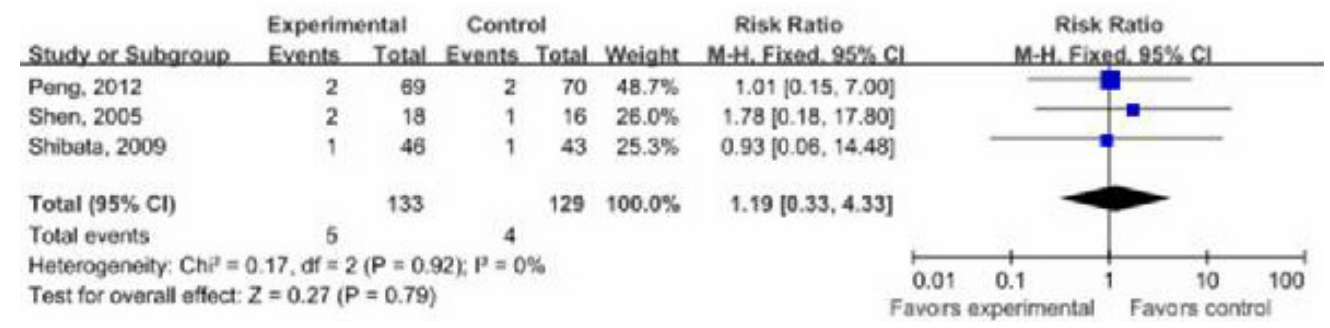

Figure 8. Forest plot of major complication rates for the experimental groups versus the control groups of the studies included.

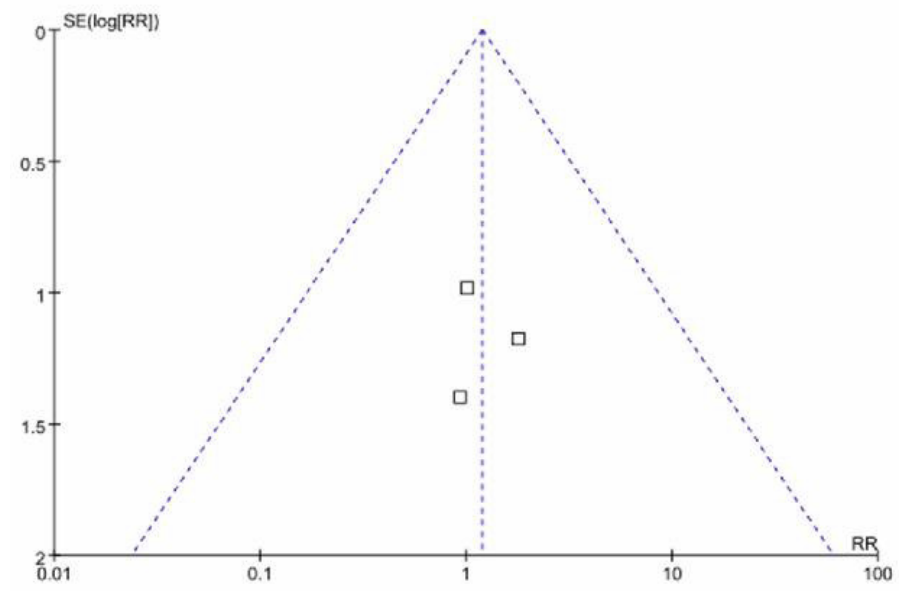

Figure 9. Funnel plot of the incidence of major complication rates in the experimental groups versus the control groups of the studies included. 


\section{Evaluation of sensitivity analysis}

We performed a sensitivity analysis by removing 1 study at a time. The above mentioned meta-analytic estimates were not changed when we re-performed the meta-analyses after excluding each study, implying that the results of the meta-analyses are reliable.

\section{DISCUSSION}

Many studies have investigated the efficacy of TACE plus RFA (experimental group) versus RFA (control group) for patients with hepatocellular carcinoma (Shen et al., 2005; Cheng et al., 2008; Yang et al., 2008; Morimoto et al., 2010). Nonetheless, the results of these studies have been confusing because they have small sample sizes or low statistical power. In this study, we retrieved 6 studies that included 531 (experimental group, 272; control group, 259) patients with hepatocellular carcinoma.

The results of our meta-analysis showed the experimental group had a higher 3-year survival rate $(\mathrm{RRs}=1.41 ; 95 \% \mathrm{Cl}=1.03-1.94 ; \mathrm{P}<0.05)$ and a higher 2 -year survival rate $(\mathrm{RRs}=1.11$; $95 \% \mathrm{Cl}=1.01-1.23 ; \mathrm{P}<0.05)$ than the control group. The overall meta-analysis of the experimental versus control groups showed overall RRs of $2.02(95 \% \mathrm{Cl}=1.40-2.91 ; \mathrm{P}<0.05)$ for the 3 -year recurrence-free survival rate and $1.63(95 \% \mathrm{Cl}=1.06-2.51 ; \mathrm{P}<0.05)$ for the 5-year recurrence-free survival rate. The overall meta-analysis also showed overall RRs of $0.75(95 \% \mathrm{Cl}=0.60-0.93 ; \mathrm{P}<$ $0.05)$ for the incidence of tumor progression and $1.19(95 \% \mathrm{Cl}=0.33-4.33 ; \mathrm{P}>0.05)$ for the major complication rate in the experimental group versus the control group. In the sensitivity analysis, the above mentioned meta-analytic estimates were unchanged by removing 1 study at a time.

Hepatitis $\mathrm{C}$ or $\mathrm{B}$ virus infection is a common cause of chronic liver disease and hepatocellular carcinoma (Pinzone et al., 2014). TACE is the mainstay treatment for patients with unresectable hepatocellular carcinoma. In patients with poor liver function, superselective catheter placement is necessary to prevent treatment-induced liver failure (Takao et al., 2007).

The degree of heterogeneity is one of the major concerns regarding the validity of a metaanalysis (Moreno et al., 2012). Publication bias is another important factor that affects the quality of a meta-analysis. Meta-analyses are susceptible to publication bias, which is a problem that occurs when there has been selective publication of studies with positive results (Niemeyer et al., 2012). Here, funnel plots were used to assess publication bias. No evidence of publication bias was observed for the comparison of the experimental and control groups when all studies were included. Moreover, we performed a sensitivity analysis by removing 1 study at a time and repeating the meta-analyses. There was little change to our meta-analytic estimates in the sensitivity analysis, implying that the meta-analysis results are reliable.

However, some limitations to the present study should also be discussed. First, significant differences in between-study heterogeneity were detected, and may have distorted the metaanalysis. However, this heterogeneity did not represent a major problem because we performed a subgroup analysis to reduce heterogeneity. In addition, the number of studies was small (6); thus, more high-quality RCTs are necessary to test and verify the results of this meta-analysis.

\section{CONCLUSION}

This meta-analysis indicated that the experimental group had a higher survival rate, 
a higher recurrence-free survival rate, and a lower incidence of tumor progression than the corresponding control group.

\section{Conflicts of interests}

The authors declare no conflict of interest.

\section{ACKNOWLEDGMENTS}

manuscript.

We are grateful to Dr. Xu for providing comments and suggesting improvements to the

\section{REFERENCES}

Archambeaud I, Auble H, Nahon P, Planche L, et al. (2014). Risk factors for hepatocellular carcinoma in Caucasian patients with non-viral cirrhosis: the importance of prior obesity. Liver Int. 35: 1872-1876.

Bianco S, Merkel C, Savastano S, Bellon S, et al. (1996). Short-term effects of transcatheter arterial chemoembolisation on metabolic activity of the liver of cirrhotic patients with hepatocellular carcinoma. Gut 39: 325-329.

Chang KC, Tseng PL, Wu YY, Hung HC, et al. (2014). A polymorphism in IFNL3 is an independent risk factor for development of hepatocellular carcinoma after treatment of HCV infection. Clin. Gastroenterol. Hepatol. 13: 1017-1024.

Cheng $B Q$, Jia CQ, Liu CT, Fan W, et al. (2008). Chemoembolization combined with radiofrequency ablation for patients with hepatocellular carcinoma larger than $3 \mathrm{~cm}$ : a randomized controlled trial. JAMA 299: 1669-1677.

DerSimonian R and Laird N (1986). Meta-analysis in clinical trials. Control. Clin. Trial 7: 177-188.

Ferreiro AO, Vazquez-Millán MA, López FS, Gutiérrez MG, et al. (2014). Everolimus-based immunosuppression in patients with hepatocellular carcinoma at high risk of recurrence after liver transplantation: a case series. Transplant. Proc. 46: 3496-3501.

Flemming JA, Kim WR and Terrault NA (2015). Reply to prediction of hepatocellular carcinoma: Using a complex risk model or assisting for smoking cessation? Cancer 121: 1150.

Hallager S and Weis N (2014). Increased risk of hepatocellular carcinoma in patients with chronic Hepatitis C. Ugeskr. Laeger 176. Higgins JP, Thompson SG, Deeks JJ and Altman DG (2003). Measuring inconsistency in meta-analyses. BMJ 327: 557-560.

Lau J, Ioannidis JP and Schmid CH (1997). Quantitative synthesis in systematic reviews. Ann. Intern. Med. 127: $820-826$.

$\mathrm{Li} \mathrm{Cl}$, Chen HJ, Lai HC, Liu CS, et al. (2015). Hyperglycemia and chronic liver diseases on risk of hepatocellular carcinoma in Chinese patients with type 2 diabetes - National Cohort of Taiwan Diabetes Study. Int. J. Cancer 136: 2668-2679.

Mantel N and Haenszel W (1959). Statistical aspects of the analysis of data from retrospective studies of disease. J. Nat. Cancer Inst. 22: 719-748.

Moreno SG, Sutton AJ, Thompson JR, Ades AE, et al. (2012). A generalized weighting regression-derived meta-analysis estimator robust to small-study effects and heterogeneity. Stat. Med. 31: 1407-1417.

Morimoto M, Numata K, Kondou M, Nozaki A, et al. (2010). Midterm outcomes in patients with intermediate-sized hepatocellular carcinoma: a randomized controlled trial for determining the efficacy of radiofrequency ablation combined with transcatheter arterial chemoembolization. Cancer 116: 5452-5460.

Niemeyer H, Musch J and Pietrowsky R (2012). Publication bias in meta-analyses of the efficacy of psychotherapeutic interventions for schizophrenia. Schizophr Res. 138: 103-112.

Pais R, Lebray P, Rousseau G, Charlotte F, et al. (2015). Nonalcoholic fatty liver disease increases the risk of hepatocellular carcinoma in patients with alcohol-associated cirrhosis awaiting liver transplants. Clin. Gastroenterol. Hepatol. 13: 992999.e2.

Peng ZW, Zhang YJ, Liang HH, Lin XJ, et al. (2012). Recurrent hepatocellular carcinoma treated with sequential transcatheter arterial chemoembolization and RF ablation versus RF ablation alone: a prospective randomized trial. Radiology 262: 689-700.

Pinzone MR, Zanghi AM, Rapisarda L, D’Agata V, et al. (2014). Cirrhotic patients are still at risk of developing hepatocellular carcinoma despite interferon-induced sustained virological response. Eur. Rev. Med. Pharm. Sci. 18: 11-15.

Ridruejo E (2014). Does hepatitis B virus therapy reduce the risk of hepatocellular carcinoma? Expert Opin. Drug Saf. 14: 439-451.

Shen SQ, Xiang JJ, Xiong CL, Wu SM, et al. (2005). Intraoperative radiofrequency thermal ablation combined with portal vein 
infusion chemotherapy and transarterial chemoembolization for unresectable HCC. Hepatogastroenterology 52: 14031407.

Shibata T, Isoda H, Hirokawa Y, Arizono S, et al. (2009). Small hepatocellular carcinoma: is radiofrequency ablation combined with transcatheter arterial chemoembolization more effective than radiofrequency ablation alone for treatment? Radiology 252: 905-913.

Shimohira M, Ogino H, Kawai T, Kushita A, et al. (2011). Use of the triaxial microcatheter method in super-selective transcatheter arterial chemoembolisation for hepatocellular carcinoma. Brit. J. Radiol. 84: 184-187.

Takao H, Doi I and Watanabe T (2007). Superselective transcatheter arterial chemoembolisation of an unresectable hepatocellular carcinoma using three-dimensional rotational angiography. Brit. J. Radiol. 80: e85-87.

Wu ZF, Zhang HB, Yang N, Zhao WC, et al. (2012). Postoperative adjuvant transcatheter arterial chemoembolisation improves survival of intrahepatic cholangiocarcinoma patients with poor prognostic factors: results of a large monocentric series. Eur. J. Surg. Oncol. 38: 602-610.

Yang P, Liang M, Zhang Y and Shen B (2008). Clinical application of a combination therapy of lentinan, multi-electrode RFA and TACE in HCC. Adv. Ther. 25: 787-794.

Zhang H, Liu C, Han YC, Ma Z, et al. (2015). Genetic variations in the one-carbon metabolism pathway genes and susceptibility to hepatocellular carcinoma risk: a case-control study. Tumour Biol. 36: 997-1002.

Zhao X, Wang T, Liu B, Wu Z, et al. (2015). Significant association between upstream transcription factor 1 rs2516839 polymorphism and hepatocellular carcinoma risk: a case-control study. Tumour Biol. 36: 2551-2558. 